\title{
Short Communication: Examining taxa representation in Asian zoos and aquaria using historic records
}

\author{
JAMES EDWARD BRERETON`, SHELBY RENEE BRERETON \\ University Centre Sparsholt, Winchester, Hampshire, England. \\ •email: James.Brereton@sparsholt.ac.uk
}

Manuscript received: 29 March 2021. Revision accepted: 26 April 2021.

\begin{abstract}
Brereton JE, Brereton SR. 2021. Short Communication: Examining taxa representation in Asian zoos and aquaria using historic records. Biodiversitas 22: 2870-2875. According to the World Association of Zoos and Aquariums (WAZA), modern zoological collections should engage in conservation and education-based activities: to do this, the collections must house animals that are interesting to their visitors. There is a plethora of evidence to suggest that zoo visitors and the wider public are more interested in mammals than any other taxa. This 'mammal bias' is known to extend into facets of science, such as in reintroduction and zoo research literature. Previous studies on zoo collection planning, however, have identified that globally, zoological collections actually contain more species of bird and fish on average than mammals. At current, there is limited information on the composition of Asian zoological collections. Data on the number of species per taxa (amphibians, birds, fish, invertebrates, mammals, and reptiles) housed at each zoo globally was collected from the International Zoo Yearbook's 'Zoos and Aquariums of the World' from 1960 until 2018. Additional data including the visitor attendance age and size of the collection was recorded. The composition of Asian zoo collection plans was compared against zoos globally, and a longitudinal investigation of Asian Zoo composition was also conducted. Additionally, Poisson regressions were run to determine whether the proportional representation of taxa, year, or visitor attendance were predictors of the numbers of animal species at the collection. Overall, the number of species per taxa significantly differed between Asian collections and the global averages, with Asian collections generally containing greater numbers of invertebrates and fish, and comparatively fewer reptiles. There were similarities in the number of species per taxa over time between Asian collections and zoological collections globally. This study suggests that the influences that affect the species housed in zoos and aquariums more globally are also influencing those in Asian countries.
\end{abstract}

Keywords: Amphibians, collection planning, fish, mammals, taxonomic bias

\section{INTRODUCTION}

Globally, zoos and aquariums are united under the "World Zoo and Aquarium Conservation Strategy" (WAZA 2005), which suggests that animal collections should engage in conversation and educated-related activities (Jensen et al. 2017). Engaging in these activities allows zoos and aquariums to engage local communities and promote the protection of biodiversity.

The animals housed by zoological facilities must therefore play multiple roles. They must be sufficiently interesting to attract an audience of paying customers into the facility (Moss and Esson 2010). However, some of the animals should also have some conservation value (for example, for conservation breeding, reintroduction or conservation research purposes) (Wood et al. 2020), and some may fit within an educational program (for example, to make visitors aware of the dangers of invasive species, or perhaps local wildlife). In a well-developed collection plan, all animals, therefore, have a purpose in the facility (Lacy 2013).

However, these purposes might not always be synergistic. For example, there is considerable evidence to suggest that zoo visitors, and the public more generally, are most interested in mammals, particularly when they are large, and active (Moss and Esson 2010; Courchamp et al. 2018). By contrast, many endangered species are small, dull in color and not mammalian (Martin et al. 2014). For example, over 50 species of Partula snail (Partula spp.) are now extinct (Phillips et al. 2020). There is a need, therefore, to consider both conservation and educational values of animals when accessioning them into collections.

Furthermore, a mammal-biased animal collection plan does not adequately reflect biodiversity, or the types of conservation issues that occur in the wild. According to the International Union for the Conservation of Nature (IUCN 2021), approximately $4.7 \%$ ( $72,906 / 1,551,844 \mathrm{spp}$.) of all extant animal species are vertebrates. There numbers may be higher still, as many invertebrate species are yet to receive a scientific name (IUCN 2021). To show animal biodiversity in a way that actually represents nature, zoological collections would consist almost entirely of invertebrate species.

It is perhaps unrealistic to expect zoos and aquariums to try to fully represent biodiversity, especially when considering the need to attract customers. Instead, collections could make sure that all major taxonomic groups are represented in the collection plan. This would 
encourage visitors to leave with a more holistic view of conservation and biodiversity, and could also contribute to the zoos pledge to education (Jensen et al. 2017).

\section{Influences on current collection plans}

Zoos cannot rapidly adjust their collection plans, and the addition or removal of animal species is often challenging. New animals might be acquired through successful breeding, trade with other collections, through confiscations, or collection from the wild (Durrell 1960). Given that collection from the wild is relatively rare for zoos currently due to regulations such as the Convention on the International Trade of Endangered Species of Fauna and Flora (CITES), animal additions may be limited. Movement between regions is also, similarly, limited. For example, importing placental mammals into Australia requires considerable planning, followed by a lengthy quarantine for the animal in question. Therefore, many of the animals that are currently held in collections are the result of historical choices and the survival and breeding of particular species.

Public interest is one of the key reasons why species might be selected for zoological collection plans. There are several themes in terms of animal interests that appear to be largely consistent across countries and cultures. For example, there seems to be much greater interest in mammalian species (Moss and Esson 2010). Generally, researchers have also demonstrated that the public is more interested in animals with larger body size (Moss and Esson 2010; Courchamp et al. 2018), that are brightly colored, active (Carr 2016). From these perspectives, one might house more mammalian megafauna in order to keep visitors interested and engaged.

Given the complex purposes of animals in the zoo or aquarium, the actual composition of zoological collection plans may not necessarily reflect the mammal bias, or conservation-oriented focus. An investigation into collection plans globally revealed that birds were actually the most commonly housed taxon in terms of average numbers of species per collection $(31.37 \%$ of the average collection plan) (Brereton and Brereton 2020). This trend, with birds being the most common taxa, had been a consistent trend across a 58-year period. By contrast, the number of species of amphibians, a conservationdependant group, were low for each collection $(2.78 \%$ of the average collection plan). There was no evidence to suggest that the average number of amphibians had increased over time, despite the new conservation crisis for the taxon (Browne et al. 2011).

One element that must be considered in animal collection planning is sustainability; the ability of an animal population to continue in captivity indefinitely (Lacy 2013). In the early $20^{\text {th }}$ Century, animal collections may not have considered sustainability as a priority, as deceased animals could have been replaced with wildcaught individuals (Durrell 1960). Now, with many wild animal populations becoming endangered or extinct, coupled with legislation, such as CITES, animal imports are rare (Lacy 2013). Sustainable breeding in captivity is therefore essential if zoos plan to show wild animals in the future.

Developing sustainable populations comes with several challenges. For example, consideration must be paid to the genetic health of the population, and this may require large numbers of individuals to be kept from each species if inbreeding is to be avoided (Lacy 2013). In turn, this places pressure on exhibit space: larger populations mean fewer exhibits available at each zoo, especially as pressure for good animal welfare places emphasis on larger exhibit sizes (Brereton 2020). This movement toward sustainability might intuitively imply that zoological collections will reduce the variety of species they keep, so as to focus on a few endangered species (Lacy 2013). This does indeed seem to be the case: zoo records suggest that the average number of species housed at the zoo has dropped from 329 in 1960 to 225 in 2018 (31.4\% decrease) (Brereton and Brereton 2020).

\section{Influences of Asian collection planning}

The major zoo organization covering Asia is the South East Asian Zoos Association (SEAZA), whose objective is to strengthen the role of conservation in zoological facilities (Agoramoorthy 2004). The challenges faced by SEAZA's members may contrast with the problems experienced in regions such as North America and Australia.

Many of the zoos and aquariums in Asia are surrounded by regions of exceptionally high biodiversity: biodiversity hotspots (Le Roux et al. 2019). Many of the species arriving in zoological collections are the result of confiscations of animals (Banes et al. 2018). Species include great apes (Banes et al. 2018), gibbons, turtles, and tortoises (Rivera et al. 2021). The accession of great numbers of new animals places pressure on zoological facilities in terms of space, resources, and staffing. Multiple confiscations may also undermine the need for conservation breeding, especially where facilities are already at capacity (Rivera et al. 2021).

Differences in culture, and also as a result of confiscations, may have a considerable impact on the species housed in Asian zoos (Banes et al. 2018). If zoos are taking in largely confiscated animals, the collection plans may actually reflect the types of species that are most commonly being taken as pets (Rivera et al. 2021). At current, the collection plans of Asian facilities have not been quantified in published literature. However, some research is available on collection planning more widely from a global perspective (Brereton and Brereton 2020). Further research on the collection plans of Asian facilities would therefore have value in determining how they compare to other regions.

\section{MATERIALS AND METHODS}

\section{Zoo collection plans}

Data were collected from the 'Zoos and Aquariums of the World' chapter entitled International Zoo Yearbook, using volumes 1 through to 52 (1960 to 2018). Not all volumes of the Yearbook included this chapter, so some 
years were omitted. See Brereton and Brereton (2020) for a full list of Yearbook volumes and their content.

Data was collected from every animal collection included within the Yearbook, except on occasions where there was no information on their animal collection (number of species per taxonomic Class.) For all collections that were included in the dataset, the collection's name, location (country and continent) and annual attendance figures were recorded. Additionally, the number of animals they held in each taxonomic Class was included. The Classes were amphibians, birds, fish, mammals and reptiles. A sixth term, invertebrates, was also used. Whilst this is not a single animal Class, but many (IUCN 2021), the zoo yearbook data lumped all invertebrates into one single category. Where available, further information was collected including the geographic size of the animal collection and the year the facility was opened.

\section{Data analysis}

Data were compiled into an Excel ${ }^{\mathrm{TM}} 2016$ spreadsheet, and the additional variable of 'year published' was added. Graphs were produced using Excel ${ }^{\mathrm{TM}}$. For data analysis, data were uploaded to Minitab, version 18.

The aim was to compare the number of species in each taxa for Asian collections to those of zoos more globally. To do this, a bar chart was developed to show the average number of species per taxa by continent. Using the most recent 2018 data only, the number of species per taxa was compared to see whether there was a significant difference between Asia and collections globally. Where the data were normally distributed, T-tests were used; otherwise, Mann Whitney $U$ tests were used for the comparisons.

Next, the effect of time on Asian collection plans was investigated. A line graph was generated to show how the average number of species per collection changed from 1960 to 2018. For comparison, a line graph was also generated showing the global averages per taxa over time. For each taxon, a correlation was run (Pearson where normally distributed, Spearman's where not normally distributed), to identify whether there was a correlation between the numbers of species per taxa in Asia and in collections globally.

Finally, tests were undertaken to determine what the best predictors of high species diversity were for Asian zoos. To do this, Poisson regression was run, using the total number of species as the dependant variable, and the visitor attendance and year that the data was collated. Additionally, the proportional representation of amphibians, birds, fish, invertebrates, mammals and reptiles were included as predictors. The proportional representation, rather than the numbers of species per taxa, was used to avoid issues with multicollinearity. The critical value for all tests was set to $\mathrm{P}<0.05$

\section{RESULTS AND DISCUSSION}

\section{Asian and global collection plan}

A graph was developed to show the differences in average number of species per taxa by continent (Figure 1). On average, Asian collections showed the highest numbers of fish and invertebrate species, and the lowest average number of reptiles.

\section{Temporal changes in the collection plan}

Graphs were developed to show how the average number of species per collection changed over time for Asian collections (Figure 2), and more globally (Figure 3). Correlations were run on the average number of animals per taxa per year for Asian collections versus collections globally (Table 1). All correlations were positive and significant, with the exception of reptiles.

\section{Predictors of biodiversity in collection plans}

The Poisson regression on total number of species per Asian collection was significant (Table 2), and the model explained $57.99 \%$ of the variation.

Table 1. Correlations between the average number of species per taxa over time in Asian collections versus global collections.

\begin{tabular}{llll}
\hline Taxon & Test & $\mathbf{r}$ & $\mathbf{P}$ \\
\hline Amphibians & Spearman's & $r_{s}(38)=0.463$ & $0.004^{*}$ \\
Birds & Spearman's & $r_{s}(38)=0.887$ & $<0.001^{*}$ \\
Fish & Spearman's & $r_{s}(38)=0.779$ & $<0.001^{*}$ \\
Invertebrates & Pearson's & $r(38)=0.875$ & $<0.001^{*}$ \\
Mammals & Spearman's & $r_{s}(38)=0.913$ & $0.001^{*}$ \\
Reptiles & Spearman's & $r_{s}(38)=0.339$ & $0.037^{*}$ \\
Total & Spearman's & $r_{s}(38)=0.204$ & 0.219 \\
\hline
\end{tabular}

Table 2. Predictors used in Poisson regression on the total number of species per Asian zoological collection.

\begin{tabular}{llll}
\hline Predictor & Coefficient & $\boldsymbol{X}^{\mathbf{2}}$ & $\mathbf{P}$ \\
\hline Zoo attendance & 0.000 & 58902.75 & $<0.001$ \\
Year & 0.001 & 11.10 & 0.001 \\
Mammal \% & -0.035 & 99459.46 & $<0.001$ \\
Bird \% & -0.014 & 22488.41 & $<0.001$ \\
Reptile \% & -0.023 & 33251.21 & $<0.001$ \\
Amphibian \% & -0.003 & 121.12 & $<0.001$ \\
Fish \% & -0.014 & 15733.03 & $<0.001$ \\
Invertebrate \% & 0.002 & 212.35 & $<0.001$ \\
\hline
\end{tabular}




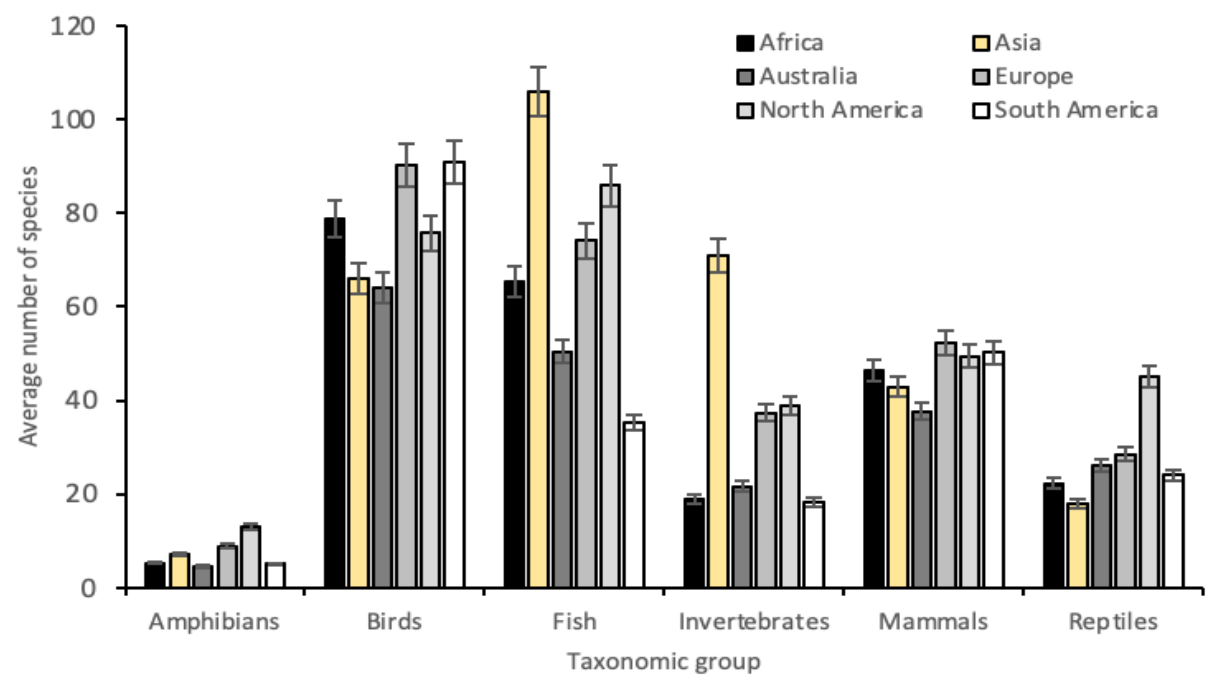

Figure 1. Average number of species per taxa per collection, separated by continent (+/- standard error)

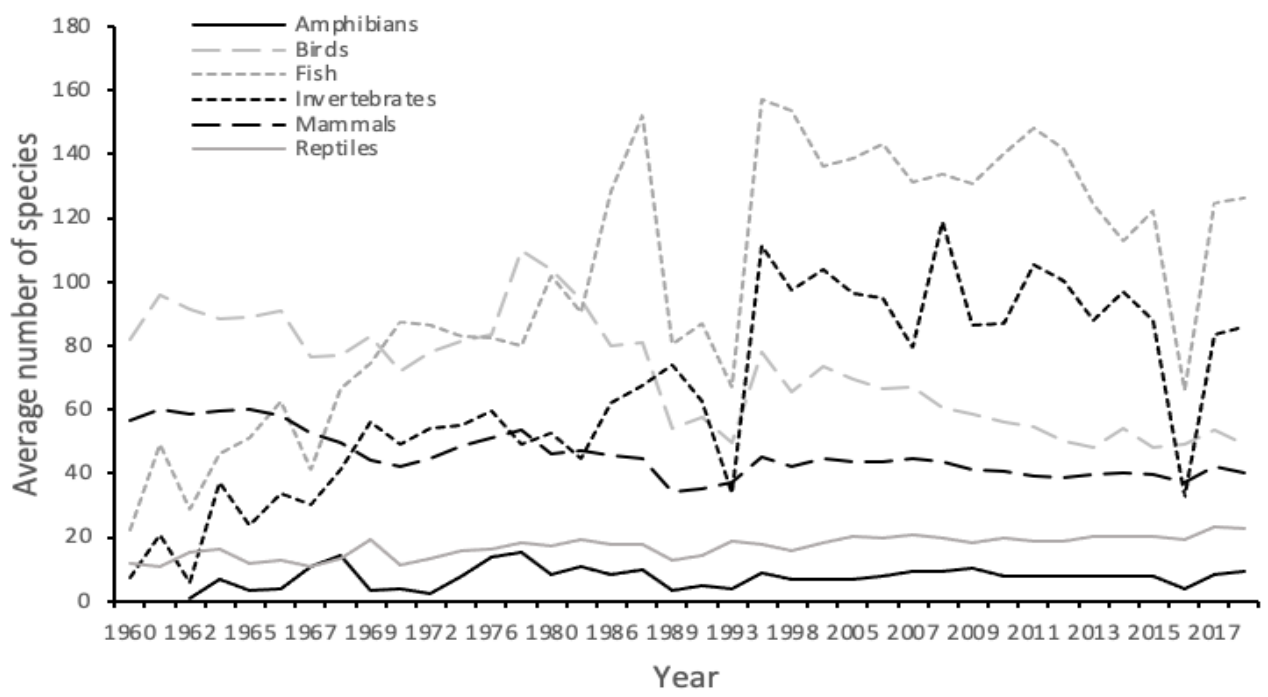

Figure 2. Average number of species per taxa per collection over time, for Asian animal collections

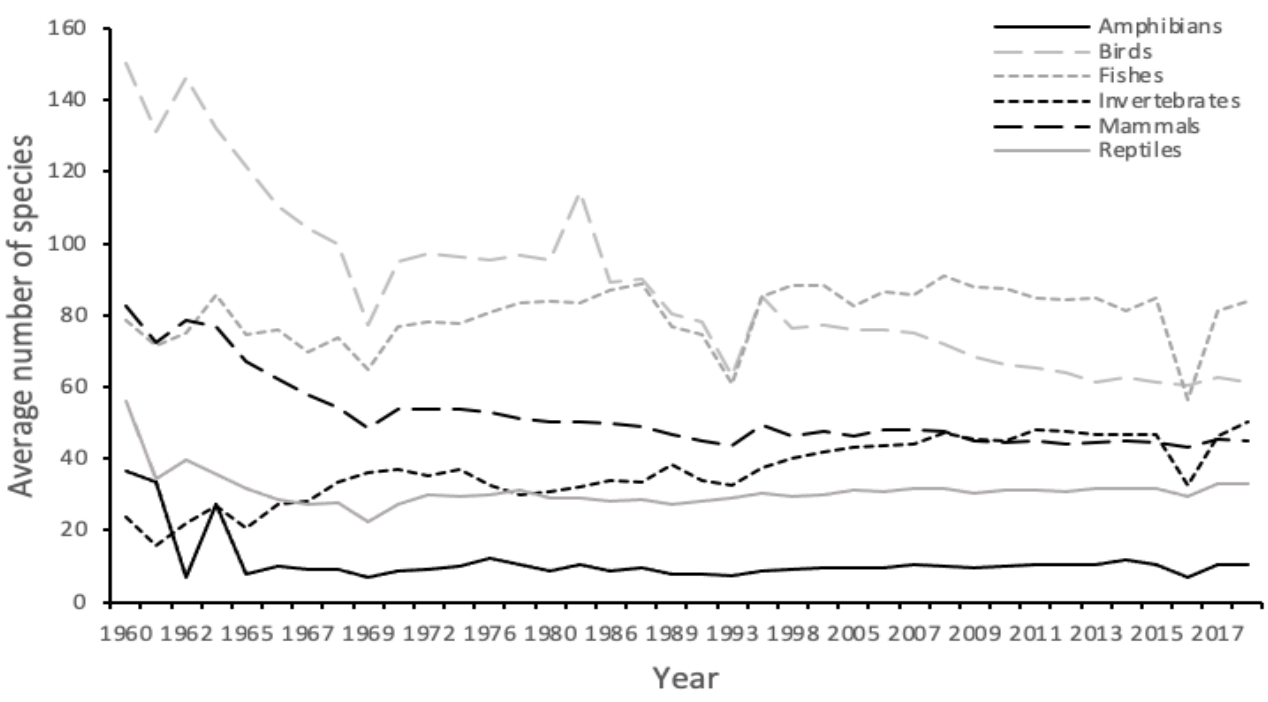

Figure 3. Average number of species per taxa per collection over time, for animal collections globally 


\section{Discussion}

Overall, this study revealed that Asian zoological collections on average contain higher numbers of invertebrates and fish, and comparatively fewer reptile species than in zoological collections in other continents. There are strong, positive correlations in terms of the changes in collection plans between Asia and the wider zoological community, suggesting that similar changes are occurring in collection plans cross-continent. A zoological collection's annual attendance and its representation of each taxonomic group was a predictor of overall number of species in the collection plan.

\section{Asian and global collection plan}

Overall, Asian collections were similar in terms of their representation of animal taxonomic classes. Two groups; fish and invertebrates, were better represented in Asian zoos than elsewhere. Fish, in terms of numbers of species, were the best represented taxonomic group in Asian collections. Partly, this may be related to availability: there are thousands of species from both taxa that are locally available (Ng and Tan 1997; Le Roux et al. 2019). Similarly, the greater prevalence of both taxa may be as a result of a history of both insect and fish-keeping in many regions of Asia (Banes et al. 2018).

It is promising to note that these taxa are well represented. This presents opportunities for zoo education to be diversified and to provide visitors with a more holistic understanding of biodiversity (Esson and Moss 2016). Exposure to a wider range of species may also promote conservation-related behaviors in the public. Interestingly, the trends in fish and invertebrate representation in Asia show a strong correlation with the changes in the representation of these taxa globally. The number of both fish and invertebrate species housed per collection in Asia and globally has risen since the 1960s. This may be as a result of improvements in knowledge of husbandry and reproductive science, and availability (Traylor-Holzer et al. 2019).

Reptiles were relatively scarce in Asian zoos in comparison to collections elsewhere. It should also be noted that while significant, the correlation between reptile representation and globally was weak $\left(r_{\mathrm{s}}=0.339\right)$. This suggests that there may be other influences on reptile keeping in Asian zoological collections. On one hand, many reptiles are available locally: of these, many are housed in private collections as pets (Kusrini et al. 2021). Despite this, there is relatively little representation of this taxonomic group. The average number of species of reptile per Asian collection remains below 20. Similarly, the number of amphibians remains low for Asian collections, despite the urgent need for amphibian ex situ conservation (Browne et al. 2011).

Mammals, despite their popularity with the general public (Courchamp et al. 2018), are not the most common taxa in Asian collections. In terms of numbers of species, fish, invertebrates and birds are actually better represented. The current data set, therefore, does not suggest that Asian collections are skewed toward the keeping of mammalian taxa only. The suggestion, therefore, that Asian zoos may be biased toward mammals due to confiscations does not, on average, appear to be true. However, it should be noted that this study assessed number of species, rather than number of individuals. Thus, a larger number of confiscated individuals from one species would not be detected in the study (Banes et al. 2018; Rivera et al. 2021).

\section{Predictors of biodiversity in collection plans}

Zoo attendance and year were both positive predictors of higher average numbers of species at Asian zoological collections. However, it is beyond the scope of this study to determine why this is the case. For example, it is possible that collections with higher visitor numbers have more funds to buy in a wider range of species and exhibits. The representation of each taxonomic group was also a predictor of the overall biodiversity of the collection. Since 1960, the average number of species per collection in both Asia and globally has dropped. This might in part be a reflection of changes in legislation (e.g. CITES) and a movement toward keeping sustainable animal populations (Lacy 2013). Similarly, movements toward larger exhibit sizes may also result in zoological collections keeping fewer animals, particularly when several small exhibits must be used to make space for one new enclosure (Whitham and Wielebnowski 2013).

\section{Limitations and future directions}

The records used in this study were compiled using the results of a survey, which historically was mailed out to zoological collections. One of the limiting factors in the study is therefore limited numbers of replies from some of the zoological collections. It should be noted, therefore, that the findings of this study aim to show trends, rather than an exact number of species per collection. Given the limited use of computer technology in the mid-twentieth century, there are limited alternatives when investigating historical trends.

Future researchers may be able to use zoo recordkeeping software, which is available online. The Zoological Information Management System (ZIMS), for example, presents an excellent opportunity to identify trends in collection planning, especially as many animal collections are already using the software or its predecessor, the Animal Record Keeping System (ARKS) (Thiruthanigesan et al. 2016). The use of this software may also allow researchers to identify trends in more detail (at taxonomic Order, Family, or Genus level).

In conclusion, Asian zoological collections, at least in terms of taxonomic representation, are similar to those in zoological collections globally. On average, Asian collections contain much higher numbers of species of fish and invertebrates than in collections elsewhere in the world. However, the trends in collection planning are generally similar to those in other countries. Whilst Asian collections may have many challenges, especially with regards to confiscations and homing of animals, this has not resulted in collections that are overtly mammal-biased in terms of numbers of species. 


\section{REFERENCES}

Agoramoorthy G. 2004. Ethics and welfare in Southeast Asian zoos. J Appl Anim Welf Sci 7: 189-195. DOI: 10.1207/s15327604jaws0703_5.

Banes GL, Chua W, Elder M, Kao J. 2018. Orang-utans Pongo spp in Asian zoos: Current status, challenges and progress towards long-term population sustainability. Int Zoo Yearb 52: 150-163. DOI: 10.1111/izy.12178.

Brereton JE. 2020. Directions in animal enclosure use studies. J Zoo Aq Res 8(1): 1-9. DOI: 10.19227/jzar.v8i1.330.

Brereton SR, Brereton JE. 2020. Sixty years of collection planning: What species do zoos and aquariums keep?. Int Zoo Yearb 54(1): 131-145. DOI: 10.1111/izy.12264.

Browne RK, Wolfram K, García G, Bagaturov MF, Pereboom, ZJJM 2011. Zoo-based amphibian research and conservation breeding programs. Amphib Reptile Conserv.5: 1-14.

Carr N. 2016. An analysis of zoo visitors' favourite and least favourite animals. Tour Manag Perspect 20: 70-76. DOI: 10.1016/j.tmp.2016.07.006.

Courchamp F, Jaric I, Albert C, Meinard Y, Ripple WJ, Chapron G. 2018 The paradoxical extinction of the most charismatic animals. PLoS Biol 16: 1-12. DOI: 10.1371/journal.pbio.2003997.

Durrell G. 1960. A Zoo in My Luggage. Penguin Books, London.

Esson M, Moss A. 2016. The challenges of evaluating conservation education across cultures. Int Zoo Yearb 50: 61-67. DOI: 10.1111/izy. 12178

IUCN. 2021

2021. Summary

Statistics. https://www.iucnredlist.org/resources/summary-statistics\#Tables_1_2

Jensen EA, Moss A, Gusset M. 2017. Quantifying long-term impact of zoo and aquarium visits on biodiversity-related learning outcomes. Zoo Biol 36: 294-297. DOI: 10.1002/zoo.21372.

Kusrini M, Palesa SP, Masy'ud B. 2021. Snake pet ownership in the city: A case study in Greater Jakarta, Indonesia. Biodiversitas 22: 1-11. DOI: $10.13057 /$ biodiv/d220424.

Lacy RC. 2013. Achieving true sustainability of zoo populations. Zoo Biol 32: 19-26. DOI: 10.1002/zoo.21029.
Le Roux JJ, Hui C, Castillo ML, Iriondo JM, Keet JH, Khapugin AA, Hirsch H. 2019. Recent anthropogenic plant extinctions differ in biodiversity hotspots and coldspots. Current Biol 29: 2912-2918. DOI: 10.1016/j.cub.2019.07.063.

Martin TE, Lurbiecki H, Joy JB, Mooers AO. 2014. Mammal and bird species held in zoos are less endemic and less threatened than their close relatives not held in zoos. Anim Cons 17: 89-96. DOI: 10.1111/acv.12069.

Moss A, Esson M. 2010. Visitor interest in zoo animals and the implications for collection planning and zoo education programmes. Zoo Biol 29: 715-731. DOI: 10.1002/zoo.20316.

$\mathrm{Ng}$ PK, Tan HH. 1997. Freshwater fishes of Southeast Asia: Potential for the aquarium fish trade and conservation issues. Aq Sci Conserv 1: 79-90. DOI: $10.1023 / \mathrm{A}: 1018335617835$

Phillips JG, Linscott TM, Rankin AM, Kraemer AC, Shoobs NF, Parent CE. 2020. Archipelago-wide patterns of colonization and speciation among an endemic radiation of Galápagos Land Snails. Heredity 111: 92-102. DOI: 10.1093/jhered/esz068.

Rivera SN, Knight A, McCulloch SP. 2021. Surviving the wildlife trade in Southeast Asia: Reforming the 'Disposal'of confiscated live animals under CITES. Animals 11: 1-20. DOI: 10.3390/ani11020439.

Thiruthanigesan K, Thiruchchelvan N, Vinitha AM, Inico JJ. 2016. Zoo Information Management System (ZIMS) for Anna Zoological Park, Chennai, India. Adv Biol Res 10: 10-14. DOI: 10.5829/idosi.abr.2016.10.1.1029.

Traylor-Holzer K, Leus K, Bauman K. 2019. Integrated Collection Assessment and Planning (ICAP) workshop: Helping zoos move toward the one plan approach. Zoo Biol 38: 95-105. DOI: 10.1002/zoo.21478

Whitham JC, Wielebnowski N. 2013. New directions for zoo animal welfare science. Appl Anim Behav Sci 147: 247-260. DOI: 10.1016/j.applanim.2013.02.004.

Wood J, Ballou JD, Callicrate T, Fant JB, Griffith MP, Kramer AT, Havens K. 2020. Applying the zoo model to conservation of threatened exceptional plant species. Conserv Biol 34: 1416-1425. DOI: $10.1111 /$ cobi.13503.

WAZA. 2005. World Zoo and Aquarium Conservation Strategy. https://www.waza.org/priorities/conservation/conservation-strategies/ 